\title{
Evaluación Comparativa del Conocimiento Sobre Salud Oral Entre Equipos Pedagógicos que Recibieron y no Recibieron Promoción en Salud
}

\author{
Comparative Evaluation of Oral Health Knowledge Among Pedagogical \\ Teams Receiving and not Receiving Health Promotion
}

\author{
Paula Caetano Araújo1; Cléa Adas Saliba Garbín²; Suzely Adas Saliba Moimaz; \\ Nemre Adas Saliba²; Diogo Reatto ${ }^{3} \&$ Renato Moreira Arcieri²
}

ARAÚJO, P. C.; GARBíN, C. A. S.; MOIMAZ, S. A. S.; SALIBA, N. A.; REATTO, D. \& ARCIERI, R. M. Evaluación comparativa del conocimiento sobre salud oral entre equipos pedagógicos que recibieron y no recibieron promoción en salud. Int. J. Odontostomat., 11(2):198-206, 2017.

RESUMEN: Este estudio tuvo como objetivo evaluar, comparativamente, las diferencias en el conocimiento sobre salud oral de equipos pedagógicos que recibieron y que no recibieron el Programa de Salud Oral, promovido por la Facultad de Odontología de Araçatuba (FOA), en las escuelas de enseñanza infantil del municipio de Araçatuba, São Paulo, Brasil. Participaron de esta investigación transversal 76 profesores de 10 escuelas municipales de enseñanza infantil (EMEB). Los equipos pedagógicos del grupo experimental recibieron las actividades del Programa de Salud Oral de la FOA ( $n=38$ ); en cambio, los equipos establecidos en el grupo de control no las recibieron $(n=37)$. Se recolectaron los datos por medio de un cuestionario que trazaba el perfil del profesor (sexo, función en la escuela, edad, nivel de escolaridad) y evaluaba su conocimiento sobre salud oral (placa dental, caries dental, cepillo de dientes, almacenamiento de cepillos, dentífrico, chupete, hábitos de higiene oral). Se compararon las variables independientes entre los grupos por medio de tests estadísticos. El $76,6 \%$ de la muestra eran de profesores de enseñanza infantil y solo uno era del sexo masculino. La media de edad de los dos grupos fue de 41 años y el grado de escolaridad fue de posgrado completo (88,1 \%). La investigación identificó comportamiento muy semejante en las respuestas del cuestionario en ambos los grupos encuestados, siendo sus respuestas muy cerca a las del sentido común. Esta investigación concluye que no hay diferencias significativas en el conocimiento sobre salud oral entre los grupos evaluados.

PALABRAS CLAVE: promoción de la salud, niños de educación infantil, salud oral.

\section{INTRODUCCIÓN}

La alta prevalencia de la caries dental y enfermedad periodontal, asociada a la lógica curativista y capitalista del sector de la salud, se relaciona directamente con los problemas de la calidad de vida del ciudadano y con el enorme gasto con procedimientos restauradores por el sistema público de salud (Silveira et al., 2014).

Se sabe que la eficacia de los tratamientos propuestos para esas enfermedades no solo dependen exclusivamente de la habilidad profesional del cirujano dentista y de su conocimiento científico sobre la terapéutica, sino también de la colaboración del paciente (Roshna \& Nandakumar, 2012). Sin embargo, la falta de profesionales habilitados, además de la alta demanda de procedimientos restauradores, perjudica la concienciación de la población en la autonomía de su salud y produce insatisfacción popular (Miccas \& Batista, 2014).

\footnotetext{
1 Facultad de Odontología, Departamento de Odontología Preventiva y Social, Universidad de Uberlândia, UFU, Brasil.

${ }^{2}$ Facultad de Odontología de Araçatuba, Departamento de Odontología Infantil y Social, Universidad Estadual Paulista "Júlio de Mesquita Filho", Unesp, SP, Brasil.

3 Máster en Administración, Gestión Humana y Social en las Organizaciones, Centro de Ciencias Sociales y Aplicadas, Universidad Presbiteriana Mackenzie - SP, Brasil.
} 
Ese descrédito con el sector de la salud impulsó transformaciones que permitieron entender que la relación salud y enfermedad no es estática y que está bajo influencia de los más diversos aspectos de la vida (Bloch et al., 2014). Se nota esa comprensión en la conceptualización de la promoción en salud, cuyas acciones de prevención de enfermedades y protección del individuo están conectadas a los aspectos sociales, económicos, políticos y culturales de la vida de las personas (Coe \& de Beyer, 2014), lo que permite la actuación intersectorial en el enfrentamiento de los problemas de salud, ya que así ellos dejan de ser responsabilidad exclusiva de un único sector (Azevedo et al., 2012).

El concepto de la promoción en salud se divulgó en la $1^{\text {a }}$ Conferencia Internacional sobre la Promoción de la Salud, en 1986, por medio de la publicación de la Carta de Ottawa (World Health Organization, 1986). Esta carta propuso cinco medidas que orientan el empleo de la promoción en salud en una colectividad: elaboración de políticas públicas en salud, creación de entornos de apoyo, fortalecimiento de acciones comunitarias, desarrollo de habilidades personales y reorientación de los servicios de salud en apoyo a la prevención de enfermedades y promoción de bienestar (World Health Organization). Desde entonces, han surgido muchas actividades en las áreas de la salud que priorizan esas medidas y que cuentan con la escuela como importante ambiente facilitador (Banfield et al., 2015).

La escuela es un ambiente que tiene un contexto muy rico para la actuación eficaz de la promoción en salud, pues cuenta con estructura física que permite al alumnado de educación infantil adquirir nuevas ideas, motivando el aprendizaje y propiciando gran número de relaciones interpersonales entre alumnos y otros sujetos (Halonen et al., 2013). Además, la escuela tiene importante papel en el desarrollo continuo de las actividades de promoción en salud (Henderson \& Rubin, 2014), ya que reúne, en el mismo ambiente, niños en edad adecuada para el aprendizaje de acciones preventivas que fortalezcan el bienestar (Tai et al., 2009).

Es en la infancia que se desarrollan los hábitos en salud (Albamonte et al., 2009). Por consiguiente, el papel de la escuela va mucho más allá de proveer educación formal a los alumnos, ya que actúa como facilitador en la creación de alternativas de autoeducación, concienciación y politización para esos futuros ciudadanos (Brazilian Education and Culture Ministry, 1998).
La promoción de salud oral asociada al ambiente escolar puede proporcionar grandes beneficios a los estudiantes y a aquellos que con ellos conviven en el cotidiano, pues estos actúan como multiplicadores de salud, cuyas acciones alcanzan, incluso, el cambio de hábitos de sus familiares (Gibbs et al., 2015). Sin embargo, para que su actuación sea efectiva, es necesario un abordaje multiprofesional que integre el equipo pedagógico de la escuela con las actividades de promoción de salud oral (Castro et al., 2014). La participación de este equipo está directamente relacionada con la motivación y el envolvimiento del alumno de educación infantil (Pommier et al., 2010).

De esta forma, la unión entre el profesional de salud oral y el profesor de educación infantil es esencial para que las actividades propuestas puedan tornarse un hábito y formar parte de la rutina de todos (Arcieri et al., 2013). Es importante destacar que el profesor está en contacto directo con el niño y ejerce gran influencia en el comportamiento del alumno de educación infantil (Silva et al., 2011).

En razón de la importancia del papel del profesor de educación infantil en las actividades de promoción de salud propuestas, esta investigación tuvo como objetivo evaluar, de forma comparativa, las diferencias en el conocimiento sobre salud oral de los equipos pedagógicos que recibieron y que no recibieron el Programa de Promoción de Salud Oral de la Facultad de Odontología de Araçatuba (FOA) en las escuelas municipales de educación infantil (EMEB) de Araçatuba.

\section{MATERIAL Y MÉTODO}

Aspectos éticos. Se aprobó esta investigación por el Comité de Ética en Investigación en Seres Humanos de la Facultad de Odontología de Araçatuba de la Universidad Estadual Paulista "Júlio de Mesquita Filho", bajo el número de protocolo FOA-283.017/2013. Se explicó el objetivo de la investigación a todos los equipos pedagógicos en las escuelas municipales de educación infantil y se recolectaron los datos por medio de un cuestionario rellenado por los propios participantes, los que debidamente firmaron el Término de Consentimiento Libre y Esclarecido antes del inicio de la recolección de los datos.

Delineamiento del estudio y características de la muestra. En 2013, se realizó un estudio observacional retrospectivo a fin de evaluar las diferencias en el co- 
nocimiento de los equipos pedagógicos que recibieron y que no recibieron las actividades del Programa de Promoción de Salud Oral de la Facultad de Odontología de Araçatuba (FOA). Los equipos pedagógicos participantes pertenecían a 10 EMEB del municipio de Araçatuba, São Paulo, Brasil. De los 85 profesionales que formaban parte de esos equipos, 76 aceptaron participar de la investigación (tasa de respuesta del $89 \%)$.

Araçatuba está situada en la región noroeste del estado de São Paulo, Brasil. Tiene una población de 191.662 habitantes (Instituto Brasileiro de Geografia e Estatística, 2014). De estos, 2.937 habitantes son matriculados en EMEB (Instituto Brasileiro de Geografia e Estatística, 2012). En 2010, el Índice de Desarrollo Humano del municipio fue 0,788 (Instituto Brasileiro de Geografia e Estatística, 2010), con un $16,2 \%$ de pobreza. Ya el Índice de Gini fue 0,47 (Instituto Brasileiro de Geografia e Estatística, 2003). La violencia en Araçatuba es mayor que en la capital del estado, con tasa de 20 homicidios por 100.000 habitantes (Waiselfisz, 2011).

Selección de las Escuelas Municipales de Educación Infantil (EMEB). El municipio de Araçatuba tiene 35 escuelas municipales de educación infantil (Instituto Brasileiro de Geografia e Estatística, 2012). El Programa de Promoción de Salud Oral de la Facultad de Odontología de Araçatuba se organiza en ciclos de visita a cinco escuelas a la vez a lo largo de un período de seis meses consecutivos. Las escuelas del grupo experimental fueron las últimas a recibir visitas en el primer semestre de 2013. Ya el grupo de control se compuso de EMEB que nunca habían recibido el programa. Se seleccionaron las escuelas del grupo de control de acuerdo con la ubicación geográfica de las escuelas del grupo experimental, o sea, para cada escuela del grupo experimental había una escuela del grupo de control localizada en el mismo barrio.

Grupos de comparación. Se formaron dos grupos: el grupo experimental, formado por equipos pedagógicos de cinco EMEB que recibieron el Programa de Promoción de Salud Oral de la FOA por un mínimo de seis meses; y el grupo de control, compuesto por equipos pedagógicos de EMEB que nunca habían recibido el programa.

Se recolectaron los datos durante las horas de trabajo pedagógico colectivo (HTPC) en 10 escuelas, de abril a agosto de 2013. Se entregó el cuestio- nario a los profesores para que lo respondieran individualmente. Un único investigador supervisó la aplicación de los cuestionarios para no permitir cualquier forma de cambio de información entre los profesores.

Estudio piloto. Se realizó un estudio piloto en dos EMEB que no participaron de la muestra y no hubo necesidad de realizarse alteraciones en el cuestionario después del estudio piloto.

Actividades de promoción de salud oral. Se llevaron a cabo las actividades de promoción en salud oral semanalmente por seis meses. Así, se realizaron 24 visitas en cada escuela del grupo experimental con duración de cerca de 90 minutos cada una de ellas.

Antes del inicio de las actividades del Programa, docentes y discentes del curso de grado en Odontología de la FOA acordaron las actividades que serían aplicadas, el abordaje de ejecución con los niños, la mejor forma de usar los materiales de apoyo y todos los temas que serían abordados con los alumnos, padres o cuidadores y el equipo pedagógico. Las reuniones se realizaron bimestralmente durante la aplicación del programa.

De inicio, los alumnos de grado y de posgrado en Odontología visitaron las escuelas para planificar las actividades de salud oral de acuerdo con un proyecto predeterminado. El objetivo de las visitas fue organizar las actividades para que se alcanzara el mayor número posible de niños. Se visitaron todas las salas de clase dentro de un mes, pues a cada visita se iba a dos salas con 15 niños cada.

Todas las visitas contaron con acciones recreativas, charlas sobre promoción de salud oral e higiene personal, y cepillado dental supervisado. Los miembros del equipo responsable por el Programa se dividieron en pequeños grupos responsables por la realización de las actividades a fin de garantizar que todos los niños recibieran todas las intervenciones previstas en el Programa (Tabla I).

Actividades recreativas. Las actividades recreativas relacionadas a la salud oral contaban con encajes geométricos, rompecabezas, rayuela, túnel de juego, cesta de la muela triste y feliz, teatro de títeres, pesca, taller de dibujo, macromodelos de peluche y juegos colectivos. Las actividades se desarrollaron bajo supervisión del profesor responsable por la sala de clase visitada. 
Charlas sobre el autocuidado oral y general. Las charlas sobre autocuidado en salud oral abordaron los temas del cepillado dental y uso del hilo dental y daban énfasis en la necesidad de orientación de los padres o cuidadores y equipo pedagógico durante la realización de esos procedimientos en el hogar y escuela, respectivamente. Las charlas también abordaron cuestiones nutricionales, hábitos de succión no nutritiva y el proceso de la caries dental. Respecto a la higiene general, los tópicos tratados fueron los cuidados con el pelo y uñas, limpieza de las manos, aseo durante la ducha diaria y cuidados con las ropas.

Cepillado dental supervisado. El cepillado dental supervisado se basó en la Técnica de Fones (Fones, 1934) y se realizó por un miembro del equipo de la FOA, quien auxiliaba un niño por vez. Para esta actividad, se obtuvo auxilio con el uso de macromodelos dentales y revelador de placa bacteriana.

La agenda escolar del niño también se usó para informar a los padres o cuidadores sobre la necesidad de cualquier tratamiento odontológico. El equipo acompañaba si el alumno identificado con necesidad de tratamiento lo había iniciado o no.

Los padres o cuidadores y los equipos pedagógicos participaron de charlas bimestrales y con duración de una hora con la finalidad de promover la salud oral. Los temas tenían relación con los niños y autocuidados en salud.

Los estudiantes de grado que participaron del Programa elaboraron y produjeron portacepillos dentales para todos los niños. Se confeccionaron los portacepillos dos veces al año. Más detalles se pueden consultar en la Tabla I.

\section{Instrumentos}

Equipo Pedagógico. El cuestionario usado para evaluar el conocimiento en salud oral de los equipos pedagógicos se basó en el estudio de Vasconcelos et al. (2001) y Santos (2009). Se recolectaron información sobre sexo, función en la escuela, edad y escolaridad. También se obtuvieron datos que evaluaban el conocimiento de los profesores sobre placa dental, caries dental, cepillos dentales, almacenamiento de cepillos, dentífrico, chupete y hábitos de higiene oral.

Análisis estadístico. Se realizó análisis estadístico descriptivo en el programa Epi Info 3.5.1. Se utilizó el test de Chi-Cuadrado para verificar diferencias estadísticamente significativas entre los grupos formados. Se estableció el nivel de significación de 0,05 en una prueba bilateral.

\section{RESULTADOS}

Participaron de esta investigación 76 profesionales que componen los equipos pedagógicos de $10 \mathrm{EMEB}$ del municipio de Araçatuba, São Paulo. Ambos los grupos tenían 38 participantes cada, siendo que el 76,6\% eran profesores, el $11,2 \%$ eran directores y el $13,2 \%$ eran coordinadores pedagógicos. La edad media de los participantes fue de 41 años.

Los educadores del grupo experimental presentaron mayor número de profesionales con posgrado completo $(92,1 \%)$ cuando se compararon con los educadores del grupo de control $(84,2 \%)$. El área de especialidad más frecuente en los dos grupos fue la de Educación $(42,1 \%)$.

Al considerar las preguntas que evaluaban los conocimientos específicos, el 89,5\% del grupo experimental afirmaron que el profesor de enseñanza infantil debe actuar como educador en salud oral; sin embargo, el 21 $\%$ de los participantes del grupo de control afirmaron que esta no es una competencia del profesor. Se destaca, aún, que el $47,4 \%$ del grupo experimental y el $26,3 \%$ del grupo de control se sienten aptos a orientar padres y alumnos sobre correcta alimentación y aseo. Cuando se les preguntó de la importancia de programas de promoción de salud oral en la escuela, la totalidad de la muestra confirmó la relevancia de la realización de estos programas.

Respecto a la cuestión sobre qué es la placa bacteriana, ambos los grupos afirmaron que la placa correspondía a restos de alimentos en los dientes $(76,3 \%)$. Se notó también que el grupo experimental y el grupo de control se comportaron de igual manera cuando se refieren a la caries dental como una enfermedad transmisible $(26,3 \%)$.

En relación con el cepillo dental, el grupo experimental $(92,1 \%)$ y el grupo de control (100 \%) reconocieron que un buen cepillo dental para niños debe poseer cerdas suaves y cabeza pequeña. Cabe destacar que, según los profesores del grupo experimental y de control, los cepillos dentales de los alumnos eran debidamente almacenados en portacepillos en solo el 10,5\% de las escuelas, y el $80,2 \%$ de los cepillos dentales eran identificados con el nombre del niño. 
ARAÚJO, P. C.; GARBíN, C. A. S.; MOIMAZ, S. A. S.; SALIBA, N. A.; REATTO, D. \& ARCIERI, R. M. Evaluación comparativa del conocimiento sobre salud oral entre equipos pedagógicos que recibieron y no recibieron promoción en salud. Int. J. Odontostomat., 11(2):198-206, 2017.

Tabla I. Actividades del Programa de Promoción de Salud Oral de la Facultad de Odontología de Araçatuba en las Escuelas Municipales de Educación Infantil y su relación con la Carta de Ottawa.

Actividades
El programa provoca un empoderamiento en
los alumnos de grado en Odontología que
participan de las actividades de promoción
en salud oral por medio de grupos de
estudio.

Se realizan visitas antes del inicio del Programa a las escuelas municipales de educación infantil.

Actividades del Programa de Promoción de Salud Ora

$$
\text { Objetivos }
$$

Discutir el abordaje adecuado a los niños.

Mostrar la mejor manera de usar los materiales de suporte.

Debatir todos los asuntos que serán tratados.

Capacitar a los alumnos de grado para las actividades en salud pública.

\section{Planificar las actividades según la Creación de entornos de apoyo.}

disponibilidad de infraestructura de las

escuelas.

Permitir que el equipo haga reflexión sobre las necesidades de cambios en el ambiente escolar.

Permitir que el equipo conozca a los

profesores y alumnos.

Reforzar lazos entre la universidad yla

comunidad.

Establecer vínculos afectivos.

\begin{tabular}{|c|c|c|}
\hline $\begin{array}{l}\text { Actividades recreativas en el contexto de la } \\
\text { promoción en salud oral para niños de } \\
\text { educación infantil. }\end{array}$ & $\begin{array}{l}\text { Desarrollar la habilidad cognitiva en los } \\
\text { niños. } \\
\text { Conducir prácticas preventivas en salud oral } \\
\text { y general. } \\
\text { Formar al niño como un ag ente multiplicador } \\
\text { de salud oral. } \\
\text { Establecer vínculos afectivos. }\end{array}$ & $\begin{array}{l}\text { Desarrollo de habilidades personales. } \\
\text { Fortal ecimiento de acciones } \\
\text { comunitarias. }\end{array}$ \\
\hline $\begin{array}{l}\text { Charlas sobre salud oral e higiene general a } \\
\text { niños de educación infantil. }\end{array}$ & $\begin{array}{l}\text { Prevenir enfermedades orales y sistémicas. } \\
\text { Incorporar prácticas preventivas y hábitos } \\
\text { saludables. } \\
\text { Establecer vínculos afectivos. }\end{array}$ & Desarrollo de habilidades personales. \\
\hline
\end{tabular}

Cepillado dental supervisado con los niños Ofrecer control de la placa dental.

Desarrollo de habilidades personales.

de educación infantil.

Prevenir enfermedades orales.

Mejorar la habilidad de cepillado.

Concienciar los niños sobre los beneficios del

buen cepillado dental.

Formar al niño como un ag ente multiplicador

de salud oral.

Establecer vínculos afectivos.

\begin{tabular}{|c|c|c|}
\hline Comunicados en la agenda escolar del niño. & $\begin{array}{l}\text { Informar a los padres o cuidadores sobre la } \\
\text { necesidad de tratamiento dental para sus } \\
\text { hijos. } \\
\text { Acompañar al niño para asegurarse de que } \\
\text { está recibiendo tratamiento odontológico. }\end{array}$ & Desarrollo de habilidades personales. \\
\hline $\begin{array}{l}\text { Confección de portacepillos de dientes por } \\
\text { los alumnos de grado. }\end{array}$ & $\begin{array}{l}\text { Dar almacenamiento adecuado a los cepillos } \\
\text { dentales. }\end{array}$ & $\begin{array}{l}\text { Desarrollo de habilidades personales. } \\
\text { Creación de entornos de apoyo. }\end{array}$ \\
\hline Charlas para el equipo pedagógico. & $\begin{array}{l}\text { Diseminar conocimiento sobre asuntos de } \\
\text { salud oral con foco en la temática infantil y } \\
\text { autocuidado. } \\
\text { Prevenir enfermedades orales y sistémicas. } \\
\text { Adoptar prácticas preventivas y hábitos } \\
\text { saludables. } \\
\text { Reforzar el vínculo de confianza entre el } \\
\text { profesor y el niño de educación infantil. }\end{array}$ & $\begin{array}{l}\text { Desarrollo de habilidades personales. } \\
\text { Fortalecimiento de acciones } \\
\text { comunitarias. }\end{array}$ \\
\hline
\end{tabular}


Se encontró discreta variación en la tasa de respuestas correctas sobre la cantidad adecuada de dentífrico con flúor para cepillado dental en niños cuando se comparó el grupo experimental $(79 \%)$ con el grupo de control $(76,3 \%)$. Los dos grupos obtuvieron, también, el mismo porcentaje de acierto sobre el uso del chupete $(97,4 \%)$; pero la media muestral de los grupos mostró que tan solo el $17,1 \%$ de los participantes afirmaron que la edad ideal para que el niño deje el chupete es entre los 3 y 4 años.

Acerca de los hábitos de higiene oral, el grupo experimental $(89,5 \%)$ y el grupo de control $(92,1 \%)$ declararon utilizar el cepillo dental y dentífrico tres o más veces al día. Se encontró la misma frecuencia (tres veces o más) para el uso del hilo dental, si bien los participantes del grupo experimental presentaron mayor porcentaje (50 \%) de utilización cuando comparados con los del grupo de control $(43,7 \%)$.
Se aplicó el test de Chi-Cuadrado (Siegel, 1975) para verificar la existencia o no de diferencias estadísticamente significativas entre las frecuencias de respuestas dadas por los equipos pedagógicos de los dos grupos. Se estableció el nivel de significación en 0,05 , en una prueba bilateral (Siegel). Sin embargo, no se encontraron valores significativos.

Se resalta que esta investigación forma parte de un proyecto de investigación que evaluó, de forma comparativa, los niños de educación infantil y sus padres o cuidadores. Se utilizaron las mismas escuelas a fin de abarcar todos los actores sociales inseridos en el ambiente escolar. Por medio del test T, se constató que el Programa de Promoción de Salud Oral de la FOA está asociado a mayor conocimiento de salud oral en niños de educación infantil $(p>0,001)$. Sin embargo, no se puede afirmar lo mismo para padres o cuidadores (Tabla II).

Tabla II. Comparación entre las puntuaciones de los grupos sobre el conocimiento de hábitos de salud oral.

\begin{tabular}{lcccc}
\hline & $\begin{array}{c}\text { Grupo } \\
\text { experimental } \\
(\mathrm{N}=93)\end{array}$ & $\begin{array}{c}\text { Grupo de control } \\
(\mathrm{N}=90)\end{array}$ & $\begin{array}{c}\text { Total } \\
(183)\end{array}$ & P valor \\
\hline $\begin{array}{l}\text { Conocimiento de los niños sobre salud } \\
\text { rall, media (DP) }\end{array}$ & $11.62(1.00)$ & $8.42(2.90)$ & $10.05(2.68)$ & $<0.001$ \\
$\begin{array}{l}\text { Conocimiento de los cuidadores sobre } \\
\text { salud oral, media (DP) }\end{array}$ & $8.09(2.53)$ & $7.90(2.51)$ & $8.00(2.51)$ & 0.534 \\
\hline
\end{tabular}

$\mathrm{P}$ valor referido al test de Mann-whitney.

Teste de Kolgomornov-Smirnov para distribución normal de ambas las puntuaciones: $\mathrm{P}<0.001$.

\section{DISCUSIÓN}

Los principales hallazgos de esta investigación indicaron que no hay diferencias estadísticamente significativas en la evaluación comparativa del conocimiento sobre salud oral de los equipos pedagógicos del grupo experimental y de control.

Este resultado es un reflejo de las dificultades señaladas en el cotidiano del magisterio (Barbosa, 2012). Los profesores, en especial aquellos que se ocupan de la educación infantil, afrontan un mercado laboral limitado, con salarios reducidos y falta de reconocimiento del profesional como alguien que moldea el comportamiento social del alumno de educación infantil (Koselski, 2014). A causa de esta realidad, la búsqueda por mejores incentivos en la jornada de trabajo hizo aumentar el número de profesionales con posgrado completo (Arantes \& Gebran, 2014), hecho que se puede observar en este estudio.

Así, se propone que los programas implantados en la escuela, a fin de promover salud, intensifiquen las actividades direccionadas al equipo pedagógico para motivarlo y sensibilizarlo sobre las metas de las intervenciones, permitiendo que se sienta parte integrante de las actividades propuestas $y$, consecuentemente, insertado en un ambiente que valoriza su fuerza de trabajo. 
Pese a que el contexto profesional y social de los profesores es conturbado, todos los participantes de la investigación consideran importante los programas de promoción de salud oral realizados en la escuela. El $89,5 \%$ de los participantes del grupo experimental afirmaron que el profesor de enseñanza infantil debe, incluso, actuar como educador en salud oral. Las investigaciones de Saliba \& Saliba (1970) e Cord et al. (2015) identificaron que los profesores son elementos esenciales para el éxito de las actividades de promoción de salud, una vez que facilitan el acercamiento al niño, y, además, son un puente entre el niño de educación infantil y sus padres o cuidadores. De esta manera, se destaca la importancia de la actuación del equipo pedagógico en las actividades de promoción de salud.

El análisis de las cuestiones que evaluaron el conocimiento en salud oral (placa dental, caries dental, restauración de dientes deciduos, cepillos dentales, dentífrico y chupete) mostró que los dos grupos obtuvieron porcentajes similares en las respuestas de las preguntas, lo que demuestra que las dificultades son las mismas presentadas por el sentido común de la población. Santos et al. (2012) constataron que el conocimiento sobre salud oral en profesores necesita solidificarse, logrando una comprensión más profunda de la temática, para que ellos puedan ejercer activamente su papel de promotores de salud oral.

Igualmente es importante destacar que los dos grupos formados para esta investigación lograron una notable tasa de respuesta al afirmar que la mayoría de los cepillos dentales de sus alumnos estaba identificada con sus nombres $(80,2 \%)$, aunque su acondicionamiento era inadecuado en la mayor parte de las escuelas. Los cepillos dentales tienen potencial de transmisibilidad e inoculación de microorganismos, sea por lesiones preexistentes, sea por la abrasión de las encías durante el proceso de higienización. Así, es importante el almacenaje correcto a fin de que se evite la contaminación cruzada (Peker et al., 2014).

Sobre el cumplimiento de hábitos de higiene oral en el cotidiano, el grupo experimental mostró una frecuencia mayor que la del grupo de control. Este resultado demuestra que, aunque no se encontraron diferencias estadísticamente significativas en la comparación de los dos grupos, el grupo experimental está mejor preparado para la práctica de buenos hábitos en salud oral, lo que sugiere que hubo una sensibilización del grupo por el Programa.
Limitaciones de la investigación. No se pueden generalizar los resultados de este estudio para otras realidades, pues se realizó la investigación en escuelas municipales de Araçatuba, São Paulo, las que se sometieron a las actividades del Programa de Promoción de Salud Oral de la FOA. Es importante destacar la necesidad de comparar los resultados de esta investigación con otros estudios de mismo objetivo en Brasil y en el extranjero.

La cantidad de alumnos de grado, posgrado y técnicos de la Facultad es determinante para la formación de los equipos, pues, si reducida, podrá ser factor limitante para el alcance de un número mayor de escuelas. Igualmente se identificaron algunos conflictos relacionados a la cooperación del educador de enseñanza infantil con el equipo de la FOA. Muchas veces, los profesores no seguían el cronograma establecido en el inicio de las actividades o se presentaban contrarios al abordaje del equipo de la Facultad. Se observó un cambio de prioridades por parte de la coordinación de algunas escuelas, lo que perjudicó la utilización de la infraestructura escolar y, por consiguiente, desmotivó a todo el equipo pedagógico.

Para el desarrollo de un entorno favorable para la promoción de salud, algunas actividades del Programa sufrieron adecuaciones a la realidad de las escuelas.

Implicaciones de la investigación. Es necesario que otros estudios evalúen la eficacia, a lo largo del tiempo, del Programa de Promoción de Salud Oral en las escuelas municipales de educación infantil de Araçatuba, São Paulo, utilizando instrumentos que mensuren la calidad de vida y clasifiquen la condición de salud oral de equipos pedagógicos participantes del Programa. Además, se debe hacer un análisis detallado de las actividades realizadas a fin de que se revelen qué prácticas más favorecen los actores sociales del estudio. Finalmente, el Programa debe actuar de forma más efectiva en la concienciación de los educadores sobre las prácticas de promoción de salud para que ellos refuercen cotidianamente las actividades desarrolladas por el equipo de la FOA y, así, motiven a los niños.

\section{CONCLUSIÓN}

No se encontraron diferencias estadísticamente significativas en la evaluación del conocimiento sobre salud oral para los dos grupos formados. 


\section{AGRADECIMIENTOS}

Los autores agradecen la Coordinación de Perfeccionamiento de Personal de Nivel Superior (CAPES) por la beca de doctorado concedida a Paula Caetano Araújo.

ARAÚJO, P. C.; GARBÍN, C. A. S.; MOIMAZ, S. A. S.; SALIBA, N. A.; REATTO, D. \& ARCIERI, R. M. Comparative evaluation of oral health knowledge among pedagogical teams receiving and not receiving health promotion. Int. J. Odontostomat., 11(2):198-206, 2017.

ABSTRACT: This study's goal was to comparatively evaluate the differences between the oral health knowledge of two teaching staff groups: those that were part of the oral health promotion programs and those that were not. Seventy six (76) teachers at ten nursery schools participated in this transversal study. The teaching staff of the intervention group were part of the activities of the "Oral Health Promotion Program in state-funded nursery schools of Araçatuba, São Paulo State" of the Araçatuba Dental School (Faculdade de Odontologia de Araçatuba - FOA/UNESP) ( $\mathrm{n}=38$ ), however, the teachers from the control group were not part of the activities $(n=37)$. The data were collected through questionnaire with the goal of identifying the educator (sex; function at the school; age; years of schooling), and to evaluate the educator's knowledge regarding oral health (dental plaque, dental caries, toothbrush, storage of toothbrushes, tooth paste, pacifier, oral hygiene habits). The independent variables were compared between the groups through statistical tests. $76.6 \%$ of the sample was of teachers of the nursery schools and only one participant was male. The average age between both groups was 41 and the average education level was of graduation $(88.1 \%)$. The research identified a very similar behavior in the questionnaire answers from both groups, and the knowledge identified on the evaluation was close to common sense. This study concluded that there were not significant differences on the oral health knowledge between the evaluated groups.

preschool.

KEY WORDS: health promotion, oral health; child,

\section{REFERENCIAS BIBLIOGRÁFICAS}

Albamonte, L. I. M. S.; Charone, S. \& Groisman, S. Analysis of the oral health content in the sciences textbooks of the elementary education's first grade. Pesq. Bras. Odontoped. Clin. Integr., 9(3):295-301, 2009.

Arantes, A. P. P. \& Gebran, R. A. O curso de pedagogia e o processo de formação do pedagogo no Brasil: Percurso histórico e marcos legais. HOLOS, 6:280-95, 2014.
Arcieri, R. M.; Rovida, T. A. S.; Lima, D. P.; Garbin, A. J. I. \& Garbin, C. A. S. Analysis of teacher knowledge of early childhood education on oral health. Educar Rev., 47:301-14, 2013.

Azevedo, E.; Pelicioni, M. C. F. \& Westphal, M. F. Intersectoral practices in public policies for health promotion. Physis, 22(4):1333-56, 2012.

Banfield, M.; McGorm, K. \& Sargent, G. Health promotion in schools: a multi-method evaluation of an Australian School Youth Health Nurse Program. B. M. C. Nurs., 14:21, 2015.

Barbosa, A. Low salaries implications on Brazilian teachers' work. Rev. Educ. Polit. Debate, 2(2):384-408, 2012.

Bloch, P.; Toft, U.; Reinbach, H. C.; Clausen, L. T.; Mikkelsen, B. E.; Poulsen, K. \& Jensen, B. B. Revitalizing the setting approach supersettings for sustainable impact in community health promotion. Int. J. Behav. Nutr. Phys. Act., 11:118, 2014.

Brazilian Education and Culture Ministry. National curriculum guidelines: third and fourth cycles of basic education; cross - $s$ sectional themes. Brasilia, Education and Culture Ministry, 1998.

Castro, L. M. C.; Rotenberg, S.; Gugelmin, S. A.; de Souza, T. S. N.; Maldonado, L. A.; Menezes, M. F. G. \& Valente, M. C. Health, health promotion and multiplying agents: concepts of health and education professionals in the city of Rio de Janeiro, Brazil. Demetra, 9(2):467-81, 2014.

Coe, G. \& de Beyer, J. The imperative for health promotion in universal health coverage. Glob. Health Sci. Pract., 2(1):10-22, 2014.

Cord, D.; Gesser, M.; Nunes, A. S. B. \& Storti, M. M. T. The meanings that professionals who work in the School Health Program (SHP) give to learning difficulties: pathologization and medicalization of school failure. Psicol. Cienc. Prof., 35(1):40-53, 2015.

Fones, C. A. Mouth Hygiene. Philadelphia, Lea \& Febiger, 1934.

Gibbs, L.; Waters, E.; Christian, B.; Gold, L.; Young, D.; de Silva, A.; Calache, H.; Gussy, M.; Watt, R.; Riggs, E.; Tadic, M.; Hall, M.; Gondal, I.; Pradel, V. \& Moore, L. Teeth Tales: a community-based child oral health promotion trial with migrant families in Australia. B. M. J. Open, 5(6):e007321, 2015.

Halonen, H.; Pesonen, P.; Seppä, L.; Peltonen, E.; Tjäderhane, L. \& Anttonen, V. Outcome of a community-based oral health promotion project on primary schoolchildren's oral hygiene habits. Int. J. Dent., 2013:485741, 2013.

Henderson, E. \& Rubin, G. A model of roles and responsibilities in oral health promotion based on perspectives of a communitybased initiative for pre-school children in the U.K. Br. Dent. J., 216(5):E11, 2014.

Instituto Brasileiro de Geografia e Estatística. IBGE 2003: Demographic Census. Brasilia, Instituto Brasileiro de Geografia e Estatística, 2013.

Instituto Brasileiro de Geografia e Estatística. IBGE 2010: Demographic Census. Brasilia, Instituto Brasileiro de Geografia e Estatística, 2010.

Instituto Brasileiro de Geografia e Estatística. IBGE 2012: demographic census. Brasilia, Instituto Brasileiro de Geografia e Estatística, 2012.

Instituto Brasileiro de Geografia e Estatística. IBGE 2014: Demographic Census. Brasilia, Instituto Brasileiro de Geografia e Estatística, 2014.

Koselski, A. C. Teacher: a career in extinction or lack of motivation? Rev. Intersaberes, 9(17):178-88, 2014.

Miccas, F. L. \& Batista, S. H. S. S. Permanent education in health: a review. Rev. Saude Publica, 48(1):170-85, 2014.

Peker, I.; Akca, G.; Sarikir, C.; Alkurt, M. T. \& Celik, I. Effectiveness of alternative methods for toothbrush disinfection: an in vitro study. Scientific. World Journal., 2014:726190, 2014.

Pommier, J.; Guével, M. R. \& Jourdan, D. Evaluation of health promotion in schools: a realistic evaluation approach using mixed methods. B. M. C. Public Health, 10:43, 2010.

Roshna, T. \& Nandakumar, K. Generalized aggressive periodontitis 
and its treatment options: case reports and review of the literature. Case Rep. Med., 2012:535321, 2012.

Saliba, N. A. \& Saliba, O. Oral health education and the primary school teacher. Estomatol. Cult., 4(1):83-104, 1970.

Santos, K. T. Oral Health Education at School: An Analysis of the Subjects Involved in the Process. Tesis. Araçatuba, Universidade Estadual Paulista, 2009.

Santos, K. T.; Garbin, A. J. I. \& Garbin, C. A. S. Oral health in schools: experience report. Rev. Ciênc. Ext., 8(1):161-9, 2012.

Siegel, S. Estatística Não-Paramétrica, para as Ciências do Comportamento. São Paulo, McGraw-Hill, 1975.

Silva, K. M.; Spinrad, T. L.; Eisenberg, N.; Sulik, M. J.; Valiente, C.; Huerta, S.; Edwards, A.; Eggum, N. D.; Kupfer, A. S.; Lonigan, C. J.; Phillips, B. M.; Wilson, S. B.; Clancy-Menchetti, J.; Landry, S. H.; Swank, P. R.; Assel, M. A.; Taylor, H. B. \& School Readiness Consortium. Relations of children's effortful control and teacherchild relationship quality to school attitudes in a low-income sample. Early Educ. Dev., 22(3):434-60, 2011.

Silveira, M. F.; Marôco, J. P.; Freire, R. S.; Martins, A. M. \& Marcopito, L. F. Impact of oral health on physical and psychosocial dimensions: an analysis using structural equation modeling. Cad. Saude Pública, 30(6):1169-82, 2014.

Tai, B. J.; Jiang, H.; Du, M. Q. \& Penq, B. Assessing the effectiveness of a school-based oral health promotion programme in Yichang City, China. Community Dent. Oral Epidemiol., 37(5):391-8, 2009.

Vasconcelos, R.; da Matta, M. L.; Pordeus, I. A. \& de Paiva, S. M. School: an important information place on oral health care for the child population. P. G. R. Pós-Grad. Rev. Fac. Odontol. São José dos Campos, 4(3):43-8, 2001.

Waiselfisz, J. J. Map of Violence 2012: the new standards of homicidal violence in Brazil. 2011. Disponible en: http://www.sangari.com/ mapadaviolencia/pdf2012/mapa2012_web.pdf

World Health Organization. Ottawa Charter for Health Promotion. Geneva, World Health Organization, 1986.

\author{
Dirección para correspondencia: \\ Renato Moreira Arcieri \\ Profesor Doctor de la Facultad de Odontología de \\ Araçatuba - FOA/UNESP \\ Calle José Bonifácio 1193 \\ Barrio Vila Mendonça, Araçatuba \\ CP: 16015-050 \\ Araçatuba - SP \\ Brasil
E-mail: rarcieri@foa.unesp.br; paulinhacaetano@hotmail.com

Recibido : 31-08-2016

Aceptado: 13-02-2017 